\title{
Performance Analysis of Dual-Hop Relay System over Ricean Fading Channels
}

\author{
Maja Delibasic, Milica Pejanovic-Djurisic, and Ramjee Prasad
}

\begin{abstract}
In this paper we present an analytical model for the performance evaluation of dual-hop amplify-andforward relay transmission over non-identical Ricean fading channels on both hops. The probability density function (PDF) and moment generating function (MGF) of total received SNR (signal-to-noise ratio) are presented as a fast converging sum. Furthermore, adequate semi-closed form expressions for average bit error rate (BER) and outage probability (OP) are derived. Numerical and simulation results confirm the validity of the proposed analytical model. Additionally, we have analyzed the impact of unbalanced SNRs of the two hops on the error performance of the considered relay system.
\end{abstract}

Keywords - amplify-and-forward, bit error rate, Ricean fading.

\section{INTRODUCTION}

$\mathrm{W}$ ITH the increasing demand for new services and applications, a significant focus is on the further development of wireless communication networks, that will provide required throughputs and energy efficiency. However, it is well known that wireless signal transmission imposes serious challenges in fulfilling these demands, due to the complex nature of a wireless radio channel. Thus, in defining the adequate technical solutions for future broadband wireless networks, all the relevant characteristics of this specific transmission medium have to be taken into account.

Relay technologies have been actively studied and considered in the standardization process of nextgeneration mobile broadband communication systems, such as LTE-Advanced and mobile WiMAX. Such a concept is based on resource sharing and coordination

Paper received February 13, 2014; revised August 8, 2014; accepted August 17, 2014. Date of publication November 15, 2014. The associate editor coordinating the review of this manuscript and approving it for publication was Prof. Aleksandar Nešković.

This paper is a revised and expanded version of the paper presented at the 21th Telecommunications Forum TELFOR 2013.

This paper is a part of the research project Advanced solutions for performance improvement of wireless cooperative e-service infrastructures funded by the Ministry of Science of Montenegro, and it is partly supported by SCOPES project Fostering Development of an ICT Centre of Excellence in Montenegro and REGPOT project Fostering innovation based research for $e$-Montenegro (ForeMont).

Maja Delibasic is with the Faculty of Electrical Engineering, University of Montenegro, Dzordza Vasingtona bb, 81000 Podgorica, Montenegro; (phone: 382-68-750535; e-mail: majai@ac.me).

Milica Pejanovic-Djurisic, is with the Faculty of Electrical Engineering, University of Montenegro, Podgorica, Montenegro; (phone: 382-20-245839, e-mail:milica@ac.me).

Ramjee Prasad, is with the Center for TeleInfrastruktur (CTIF), Aalborg University, Denmark. among units of wireless network, improving system coverage and overall throughput. One or more intermediate nodes (relays - R) intervene in the communication between a source (S) and a destination (D), improving wireless link performance and increasing network coverage by splitting the communication link from the source to the destination into several shorter links/hops [1]-[3]. R terminal usually performs one of the two main relaying methods [2]: Amplify-and-Forward (AF) or Decode-and-Forward (DF). In the AF relaying mode, the relay amplifies the received signal, and without performing any decoding retransmits a scaled replica of the received signal to $D$. Depending on the source-relay channel information, $\mathrm{R}$ can perform a fixed gain (FG), also called average power scaling [2], or a variable gain (VG), or instantaneous power scaling [4].

In this paper, we assume an AF VG relay system. Such a scenario is widely explored in literature in the Rayleigh and Nakagami- $m$ fading environments [3], [5], [6]. Also, authors in [7] considered its performance in mixed Rayleigh/Rice fading channels. However, the performance analysis of these relay systems for non-identical Ricean fading channels has not received much attention, despite the importance of the Ricean model. Only a few works have analyzed the performance of relays under line-ofsight fading conditions [8], [9], mainly due to the rather complex form of Ricean distribution.

Motivated by the importance of Ricean fading, that is commonly used to describe line-of-sight (LoS) communication in micro/macro cellular systems, as well as indoor radio channels [10], in this paper we analyze a dual-hop AF relay system assuming non-identical Ricean fading on SR and RD link. Thus, we present a complete analytical model leading towards the derivation of a semiclosed form PDF (probability density function) and MGF (moment generating function) of the total received SNR (signal-to-noise ratio) at $\mathrm{D}$, for dual-hop VG AF relay transmission. Furthermore, we derive adequate expressions for the outage probability (OP) of the considered system and BER (bit error rate), in the case of DPSK (differentially phase shift keying) mapping of the input data.

The remainder of the paper is organized as follows: in Section II, the system model is presented. Channel model is described in detail, and PDF and MGF of the total received SNR are derived. Section III presents a system performance analysis, while the obtained results are presented in Section IV. Conclusions are given in Section $\mathrm{V}$ of the paper. 


\section{SYSTEM MODEL}

In this paper we analyze a dual-hop AF relay system operating in the Ricean fading environment. A source node $(\mathrm{S})$ communicates to a destination node (D) through one intermediate relay node $(\mathrm{R})$, as illustrated in Fig. $1 . \mathrm{S}$ transmits a data signal to $\mathrm{R}$ in the first time slot, and then $\mathrm{R}$ amplifies the received signal and transmits it to $\mathrm{D}$ in the second time slot. A variable gain AF is assumed, meaning that the relay station permanently estimates the SR link and, depending on the channel state information, determines the level of signal scaling applied. In this case, the total received SNR at D, $\gamma_{t}$, is obtained as [5]

$$
\gamma_{t}=\frac{\gamma_{1} \gamma_{2}}{c+\gamma_{1}+\gamma_{2}}
$$

with $\gamma_{1}$ and $\gamma_{2}$ representing the SNRs of the SR and RD link, respectively. The exact $\gamma_{t}$ is given by substituting $c=1$, while it is well approximated for $c=0$. Without loss of generality, in the further analysis we will assume that $c=0$ [5].

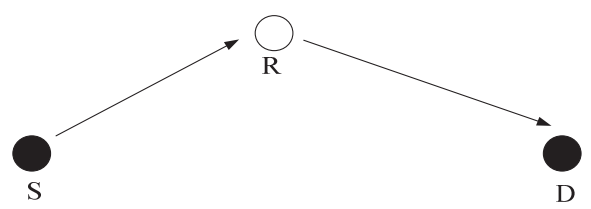

Fig. 1. Dual-hop relay system.

Assuming that both links are statistically independent, with not necessary identical Ricean fadings, the probability density function (PDF) of the instantaneous SNR of the $i$-th link is given by [10]

$$
p\left(\gamma_{i}\right)=\frac{\left(1+K_{i}\right) e^{-K_{i}}}{\bar{\gamma}_{i}} e^{-\frac{1+K_{i}}{\bar{\gamma}_{i}} \gamma} I_{0}\left(2 \sqrt{\frac{K_{i}\left(1+K_{i}\right) \gamma}{\overline{\gamma_{i}}}}\right) .
$$

$K_{i}$ denotes the Ricean $K$ factor and $\overline{\gamma_{i}}$ is the average SNR of the $i$-th link, $i=1,2$. $I_{0}($.$) is the zero order modified$ Bessel function of the first kind.

In the case that there is no $\operatorname{LoS}$ component, i.e. $K_{i}=0$, PDF of the instantaneous SNR of the $i$-th link, given in (2), becomes:

$$
p\left(\gamma_{i}\right)=\frac{1}{\gamma_{i}} e^{-\frac{\gamma_{i}}{\bar{\gamma}_{i}}} .
$$

That is a special case of Rayleigh fading distribution.

\section{A. Probability density function}

In order to determine the PDF of the total received SNR at $\mathrm{D}$, the following relation can be used

$$
p_{t}(\gamma)=\int_{-\infty}^{\infty} p\left(\gamma_{1}\right) p\left(\gamma_{2}\right) \gamma_{1 \gamma_{t}}^{\prime} d \gamma_{2} \text {. }
$$

$p\left(\gamma_{i}\right), i=1,2$, represents the PDF of the SNR of the first and the second hop, while $\gamma_{1 \gamma_{t}}^{\prime}$ is the first derivative of the SNR with respect to the total SNR. After some straightforward mathematical transformations, and an adequate change of variables, PDF given in (4) can be rewritten as

$$
\begin{aligned}
& p(\gamma)=a_{1} a_{2} e^{-\left(K_{1}+K_{2}\right)} \int_{0}^{\infty} e^{-a_{1}\left(\frac{\gamma^{2}}{w}+\gamma\right)} e^{-a_{2}(w+\gamma)}\left(\frac{\gamma+w}{w}\right)^{2} . \\
& \cdot I_{0}\left(2 \sqrt{K_{1} a_{1} \gamma\left(\frac{\gamma+w}{w}\right)}\right) I_{0}\left(2 \sqrt{K_{2} a_{2}(\gamma+w)}\right) d w
\end{aligned}
$$

with $a_{i}=\left(1+K_{i}\right) / \overline{\gamma_{i}}$, and the index $t$ for the total received SNR omitted. Using the sum representation of $I_{0}$ [11, Eq. (8.447.1)] PDF can finally be derived as in [12]

$$
\begin{aligned}
& p(\gamma)=2 a_{1} a_{2} e^{-\left(K_{1}+K_{2}\right)} e^{-\left(a_{1}+a_{2}\right) \gamma} \sum_{n=0}^{\infty} \sum_{m=0}^{n} \frac{\left(a_{1} K_{1}\right)^{m}\left(a_{2} K_{2}\right)^{n-m}}{(m !)^{2}((n-m) !)^{2}} . \\
& \gamma^{n+1} \sum_{k=0}^{n+2}\left(\begin{array}{c}
n+2 \\
k
\end{array}\right)\left(\frac{a_{1}}{a_{2}}\right)^{\frac{n-m-k+1}{2}} K_{n-m-k+1}\left(2 \gamma \sqrt{a_{1} a_{2}}\right)
\end{aligned}
$$

$K_{v}$ (.) is the modified Bessel function of the second kind. Although PDF is represented as an infinite summation, it converges fast, giving a highly accurate model with only a few terms of a sum. Here, we also examine the

\begin{tabular}{|c|c|c|c|c|}
\hline \multirow{2}{*}{$\begin{array}{l}\bar{\gamma}_{t} \\
{[\mathbf{d B}]}\end{array}$} & \multicolumn{2}{|c|}{$K_{1}=K_{2}=0 \mathrm{~dB}$} & \multirow{2}{*}{$\begin{array}{l}K_{1}=0 \mathrm{~dB} \\
K_{2}=0 \\
X=0 \mathrm{~dB}\end{array}$} & \multirow{2}{*}{$\begin{array}{l}K_{1}=5 \mathrm{~dB} \\
K_{2}=0 \mathrm{~dB} \\
X=3 \mathrm{~dB}\end{array}$} \\
\hline & $X=0 \mathrm{~dB}$ & $X=3 \mathrm{~dB}$ & & \\
\hline-5 & 9 & 8 & 5 & 12 \\
\hline-2.5 & 9 & 10 & 7 & 14 \\
\hline 0 & 9 & 11 & 7 & 15 \\
\hline 2.5 & 12 & 14 & 8 & 19 \\
\hline 5 & 15 & 16 & 8 & 23 \\
\hline
\end{tabular}
convergence rate of (6). The minimum number of terms $n$, which guarantees a four-significant-figure accuracy, is presented in Table 1. It is clear from these results that only a relatively small number of terms are necessary to achieve an excellent accuracy.

TABLE 1: THE NUMBER OF SUMMATION TERMS NEEDED FOR FOUR-FIGURE ACCURACY OF PDF, $\bar{\gamma}_{1}=0 \mathrm{~dB}$

It can also be seen that in the case of non LoS communication scenario, i.e. $K_{i}=0$ (Rayleigh), the PDF of the total received SNR becomes

$p(\gamma)=\frac{2 \gamma}{\bar{\gamma}_{1} \bar{\gamma}_{2}} e^{-\left(\frac{\gamma}{\bar{\gamma}_{1}}+\frac{\gamma}{\bar{\gamma}_{2}}\right)}\left[\frac{\left(\bar{\gamma}_{1}+\bar{\gamma}_{2}\right)}{\sqrt{\bar{\gamma}_{1} \bar{\gamma}_{2}}} K_{1}\left(\frac{2 \gamma}{\sqrt{\bar{\gamma}_{1} \bar{\gamma}_{2}}}\right)+2 K_{0}\left(\frac{2 \gamma}{\sqrt{\bar{\gamma}_{1} \bar{\gamma}_{2}}}\right)\right] .(7)$

For $K_{i}=0$, the expression in (6) is defined only if $n=0$ (the exact PDF is obtained with only one summation term). The obtained result in (7) coincides with the wellknown expression for the PDF of the total received SNR of the dual-hop AF VG system operating in the Rayleigh fading environment [13].

Fig. 2 presents the analytically obtained PDF of the total received SNR, for several values of Ricean $K$ factor per diversity branch and power imbalance between the average SNR per hop $\left(X=\bar{\gamma}_{1} / \bar{\gamma}_{2}\right)$. In order to prove the validity of the proposed analytical model, the exact PDFs obtained numerically (lines) directly from (4) are also given. 


$$
\begin{aligned}
M G F(s) & =2 a_{1} a_{2} e^{-\left(K_{1}+K_{2}\right)} \sum_{n=0}^{\infty} \sum_{m=0}^{n} \frac{K_{1}{ }^{m} K_{2}{ }^{n-m}}{(m !)^{2}((n-m) !)^{2}} \sum_{k=0}^{n+2}\left(\begin{array}{c}
n+2 \\
k
\end{array}\right) a_{1}^{\frac{n+m-k+1}{2}} a_{2} \frac{n-m+k-1}{2} \int_{0}^{\infty} e^{-\left(a_{1}+a_{2}-s\right) \gamma} \gamma^{n+1} K_{n-m-k+1}\left(2 \gamma \sqrt{a_{1} a_{2}}\right) d \gamma \\
M G F(s) & =a_{1} a_{2} e^{-\left(K_{1}+K_{2}\right)} \sum_{n=0}^{\infty} \sum_{m=0}^{n} \frac{K_{1}^{m} K_{2}^{n-m}}{(m !)^{2}((n-m) !)^{2}} \sum_{k=0}^{n+2}\left(\begin{array}{c}
n+2 \\
k
\end{array}\right) a_{1}^{n-k+1} a_{2}^{n-m} \frac{\sqrt{\pi} 2^{2 n-2 m-2 k+3}}{\left(a_{1}+a_{2}+2 \sqrt{a_{1} a_{2}}-s\right)^{2 n-m-k+3}} \frac{\Gamma(2 n-m-k+3) \Gamma(m+k+1)}{\Gamma(n+5 / 2)} \\
& \cdot F\left(2 n-m-k+3, n-m-k+\frac{3}{2} ; n+\frac{5}{2} ; \frac{a_{1}+a_{2}-2 \sqrt{a_{1} a_{2}}-s}{a_{1}+a_{2}+2 \sqrt{a_{1} a_{2}}-s}\right)
\end{aligned}
$$

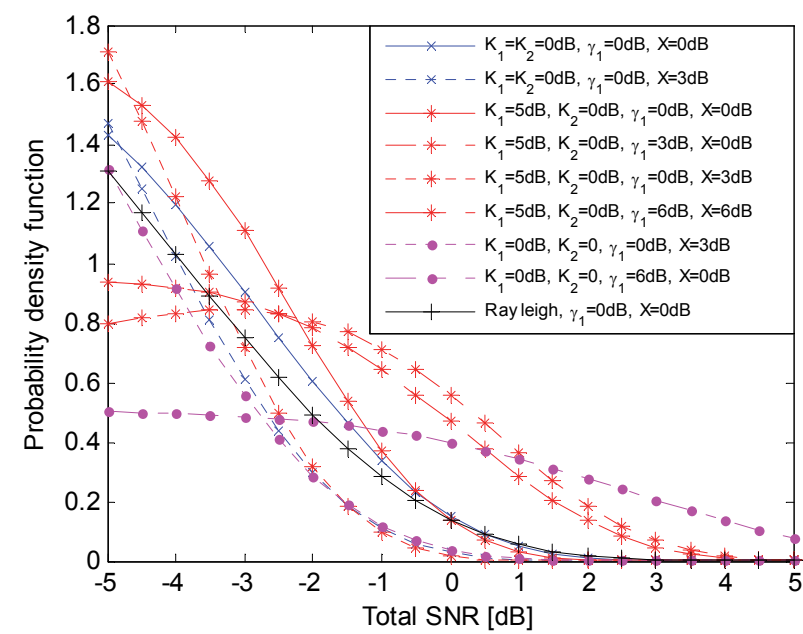

Fig. 2. Probability density function of total received SNR; lines - numerically obtained results, marker - eq. (5).

\section{B. Moment Generating Function}

The MGF is defined as the Laplace transform of PDF, i.e. $M G F(s)=\int_{0}^{\infty} p(\gamma) e^{s \gamma} d \gamma$. Using the derived PDF of the total received SNR, the MGF can be obtained as in (8). The above integral is defined in [11, Eq. (6.621.3)], thus after performing some straightforward mathematical transformations, the MGF expression is derived and given as in (9), where $F(., . ; . ;$.$) denotes the Gauss hypergeometric$ function [11, eq. (9.100)].

\section{Performance AnAlysis}

In this paper we analyze two main criteria for the performance of the system considered: outage probability and bit error rate.

\section{A. Outage probability}

Outage probability is an important performance measure used to characterize a wireless communication system. It is defined as the probability that the total instantaneous SNR falls below a predetermined threshold $\gamma_{t h}$. It can be derived as

$$
F_{\gamma}(\gamma)=\operatorname{Pr}\left[\frac{\gamma_{1} \gamma_{2}}{\gamma_{1}+\gamma_{2}}<\gamma\right]=\operatorname{Pr}\left[\gamma_{1}<\frac{\gamma \gamma_{2}}{\gamma_{2}-\gamma}\right]
$$

Averaging (10) over the distribution of $\gamma_{2}$, and applying a change of variable as in the case of PDF, OP can be found as

$$
F_{\gamma}(\gamma)=\int_{0}^{\infty} F_{\gamma_{1}}\left(\frac{\gamma(\gamma+w)}{w}\right) p_{2}(\gamma+w) d w
$$

Using a similar approach as for the PDF derivation, we obtain the following OP expression, [12]

$$
\begin{aligned}
& F_{\gamma}(\gamma)=1-2 a_{2} e^{-\left(K_{1}+K_{2}\right)} e^{-\left(a_{1}+a_{2}\right) \gamma} \sum_{k=0}^{\infty} \sum_{n=0}^{\infty} \sum_{m=0}^{n} \frac{K_{1}^{m+k} K_{2}^{n-m}}{m !(k+m) !} . \\
& \cdot \frac{a_{1}^{m} a_{2}^{n-m}}{((n-m) !)^{2}} \sum_{r=0}^{n}\left(\begin{array}{l}
n \\
r
\end{array}\right) \gamma^{n+1}\left(\frac{a_{1}}{a_{2}}\right)^{\frac{r-m+1}{2}} K_{r-m+1}\left(2 \gamma \sqrt{a_{1} a_{2}}\right)
\end{aligned}
$$

For the special case of $K_{1}=K_{2}=0$ (theRayleigh fading), the OP is defined only for $k=n=0$, thus becoming

$$
F_{\gamma}(\gamma)=1-\frac{2 \gamma}{\sqrt{\bar{\gamma}_{1} \bar{\gamma}_{2}}} e^{-\left(\frac{\gamma}{\bar{\gamma}_{1}}+\frac{\gamma}{\bar{\gamma}_{2}}\right)} K_{1}\left(\frac{2 \gamma}{\sqrt{\bar{\gamma}_{1} \bar{\gamma}_{2}}}\right)
$$

\section{B. Bit error rate}

Average bit error rate (BER) is a useful criterion for evaluating the performance of wireless communication systems. Traditionally, BER can be computed either using a MGF based approach, or averaging the conditional bit error probability over SNR distribution [10]. In this paper we consider DPSK (differentially phase shift keying) mapping. In that case we will use the MGF approach for BER determination. BER of DPSK modulated signal can be obtained as

$$
P_{b}=0.5 M G F(1)
$$

Since the MGF is derived in (9), BER can easily be determined using the resulting MGF, substituting $s=1$.

\section{RESUlts}

In this section, we present some numerical results in order to illustrate our theoretical analysis described above. These results are given for dual-hop AF VG relay systems exposed to independent and non-identical Ricean fadings, with no direct path between the source and the destination.

Fig. 3 shows the outage probability for the case of equal fading parameters (average SNR and $K$ factor) per hop. Different $K$ factors are considered, ranging from $K=0$ (the Rayleigh fading) to $K=10 \mathrm{~dB}$. The threshold value of $0 \mathrm{~dB}$ is assumed $\left(\gamma_{t h}=0 \mathrm{~dB}\right)$. As expected, it is obvious that a better OP is achieved as the Ricean $K$ factor increases. To be more precise, in order to achieve an OP of 0.1 , in the Rayleigh fading case, an average SNR per hop of $13.365 \mathrm{~dB}$ is needed. If the LoS exists between $\mathrm{S}$ and $\mathrm{R}$, and $\mathrm{R}$ and $\mathrm{D}$, such that $K$ factors of both hops have values of $0 \mathrm{~dB}$, an average SNR per hop of $12.18 \mathrm{~dB}$ is needed for the same OP. If $K_{1}=K_{2}=5 \mathrm{~dB}$, an average SNR per hop, for achieving OP of 0.1 , is $8.714 \mathrm{~dB}$, while for a strong $\mathrm{LoS}$ scenario $\left(K_{1}=K_{2}=10 \mathrm{~dB}\right)$, a SNR of $5.597 \mathrm{~dB}$ is needed. In order to achieve an OP of $10^{-2}$, average SNRs per hop of 
$23 \mathrm{~dB}, 21.78 \mathrm{~dB}, 16.2 \mathrm{~dB}$ and $8.175 \mathrm{~dB}$ are needed, in the case of Rayleigh, and Ricean fading channels having $K_{1}=K_{2}=0 \mathrm{~dB}, 5 \mathrm{~dB}$ and $10 \mathrm{~dB}$, respectively.

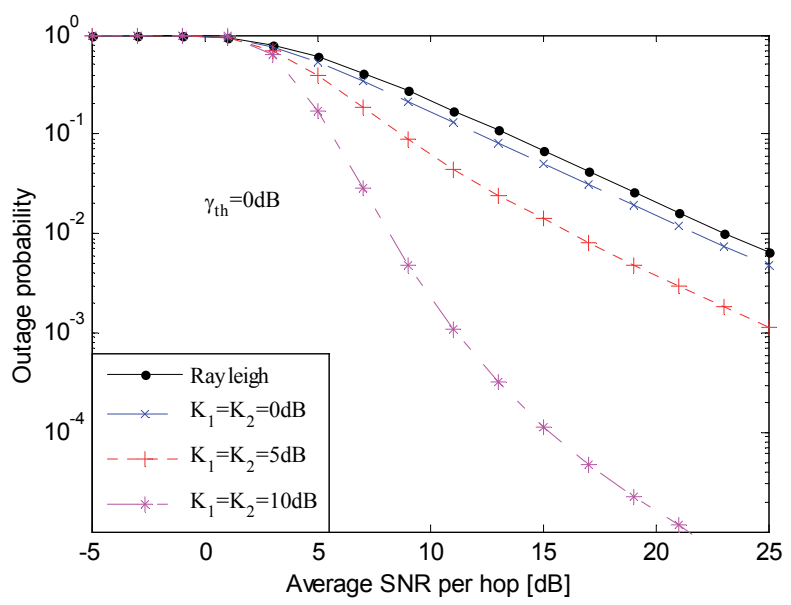

Fig. 3. Outage probability of dual-hop relay system for various $K$ factors, $\overline{\gamma_{1}}=\overline{\gamma_{2}}$.

Fig. 4 illustrates the case of non-identical fading parameters on SR and RD links, having unbalanced average SNRs per hop. In this case of non-identical fading characteristics on the two hops, slightly higher average SNRs are needed to achieve the same OP performances than in the identical fading channels on both hops. That is, in the case of mixed Rayleigh/Rice fading channels, having $K=0 \mathrm{~dB}$ on one hop, average SNRs of $12.785 \mathrm{~dB}$ per hop are needed for achieving an OP of 0.1 ; in the case when the average SNR on the first hop is $3 \mathrm{~dB}$ higher than the average SNR on the second hop, a SNR of $14.645 \mathrm{~dB}$ is needed, while for the SNRs difference of $6 \mathrm{~dB}$, a SNR of $16.868 \mathrm{~dB}$ is needed for the same OP value.

The obtained OP results indicate that the existence of LoS between $\mathrm{S}$ and $\mathrm{R}$, and/or R and $\mathrm{D}$ can significantly influence the OP performance of dual-hop relay system.

Here as well, higher $K$ factors lead to a better system performance. The presented results also show that OP increases when the SNR unbalance between SR and RD link is increased.

Another important performance measure of a wireless system is its bit error rate. Figs. 5-7 show the average BER for various $K$ factors, when DPSK modulation of the transmitted signal is implemented. In order to verify the calculated analytical results the appropriate simulation model is developed. The BER results obtained by Monte Carlo simulation are then compared to the results obtained numerically.

First, we assume equal fading parameters (average SNR and $K$ factor) per hop. In order to have a better insight into the performance improvements due to the existence of LoS, we have also plotted BER results in the case of the Rayleigh fading on both hops. It can be seen that a LoS scenario can provide a significant SNR gain. I.e., in order to achieve the same BER value, a lower SNR per hop is needed in the LoS scenario compared to the NLoS. For example, in order to achieve a BER of $10^{-1}$, in the Rayleigh fading scenario, an average SNR per fop of approximately $10 \mathrm{~dB}$ is needed, while the same BER can be achieved with an $1 \mathrm{~dB}$ lower average SNR per hop in the case when $K_{1}=K_{2}=0 \mathrm{~dB}$. In the Ricean fading scenario with $K_{1}=K_{2}=5 \mathrm{~dB}$ an almost $3 \mathrm{~dB}$ lower SNR is needed, while in a strong LoS scenario $\left(K_{1}=K_{2}=10 \mathrm{~dB}\right)$ approximately $4.3 \mathrm{~dB}$ gain is provided, compared to the NLoS scenario. For achieving the BER of $10^{-2}$, an average SNR per hop of $20 \mathrm{~dB}$ is needed, when the considered dual-hop AF VG relay system operates in the Rayleigh fading environment. A SNR gain of approximately $1.1 \mathrm{~dB}$, $5.56 \mathrm{~dB}$ and $9.65 \mathrm{~dB}$ is achieved (compared to the Rayleigh fading case) when $K$ factors on both hops are $0 \mathrm{~dB}, 5 \mathrm{~dB}$ and $10 \mathrm{~dB}$, respectively (Fig. 5).

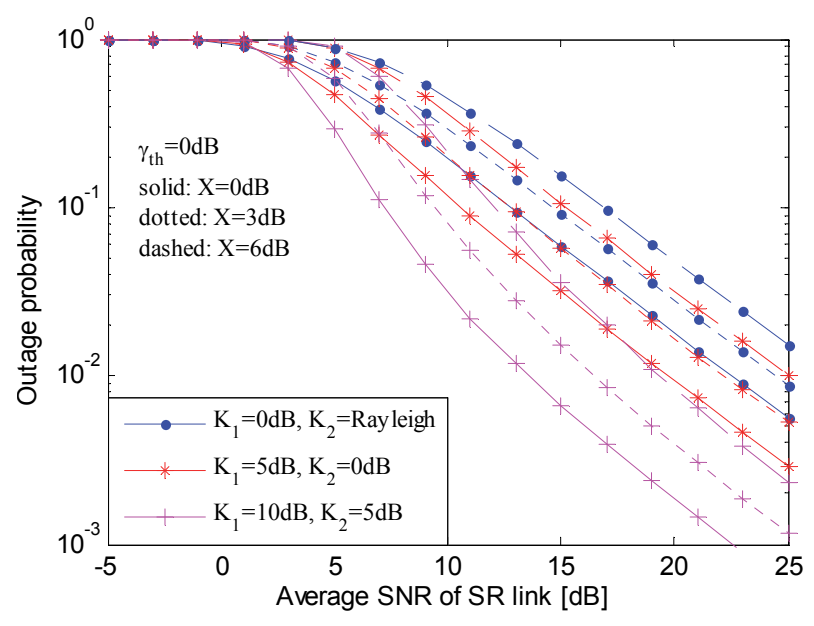

Fig. 4. Outage probability of relay system for various $K$ factors, non-identical channels.

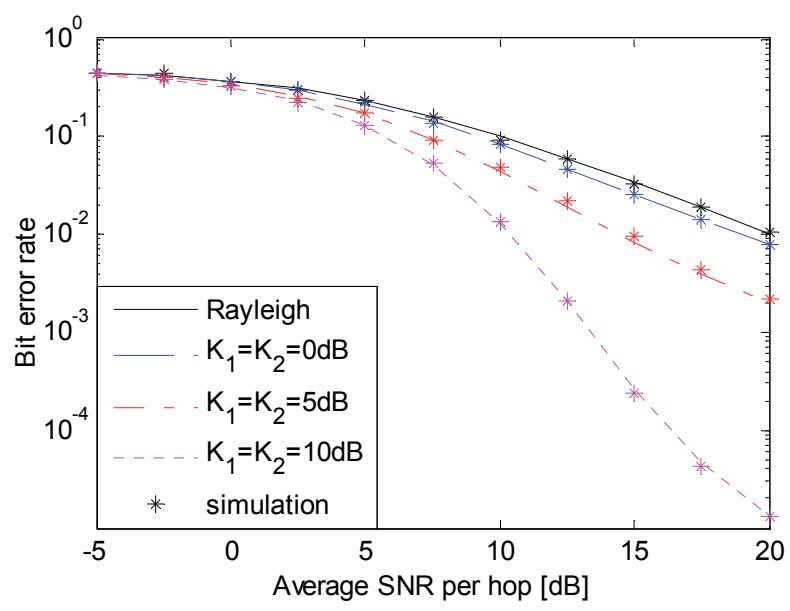

Fig. 5. BER of relay system for various $K$ factors, identical channels.

In the case of non-equal average SNRs per hop, slightly worse BER values are obtained, as illustrated in Fig. 6. For a BER of $10^{-1}$, in the case when the average SNR on the SR link is $3 \mathrm{~dB}$ higher than on the RD link, an approximately 1.6dB higher SNR per hop is needed for the same BER, while in the case when the average SNR on the SR link is $6 \mathrm{~dB}$ higher than on the RD link, an approximately $3.75 \mathrm{~dB}$ higher SNR per hop is needed for the same BER. For a BER of $10^{-2}$, the approximately $1.85 \mathrm{~dB}$ (for $3 \mathrm{~dB}$ SNRs difference), and $4 \mathrm{~dB}$ (for $6 \mathrm{~dB}$ SNRs difference) higher SNR per hop is needed. 


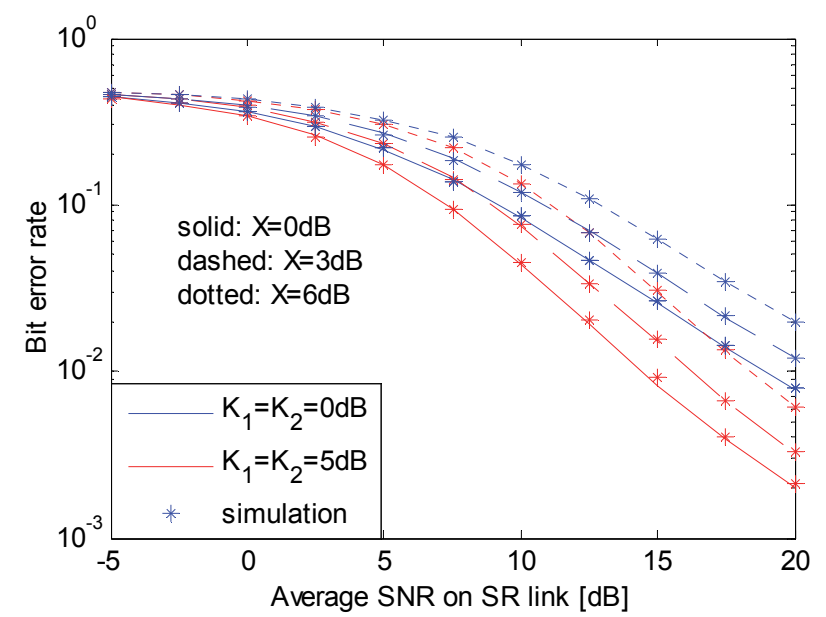

Fig. 6. BER of relay system for various $K$ factors, nonidentical SNRs.

Further on, we have also considered the scenario with non-identical fading parameters on SR and RD hops. The obtained results are presented in Fig. 7.

It is worth mentioning that although the analytical BER expression assumes an infinite summation, the results are obtained using only 20 summations for most of the considered $K$ values. As $K$ becomes higher, more summation terms are needed. The accuracy of the derived analytical model using only a few terms of an infinite sum is verified with Monte Carlo simulation. The exact match between the analytical and simulation results confirms the validity of the proposed model.

Analyzing the results given in Figs. 3-7, it is clear that the high SNR unbalance between the SR link and the RD link can significantly degrade the BER performance of the considered dual-hop relay system. Also, as the Ricean $K$ factor increases, better BER performances are achieved. In other words, if strong LoS exists between $\mathrm{S}$ and $\mathrm{R}$, as well as between $\mathrm{R}$ and $\mathrm{D}$, a high $K$ factor can provide a significant SNR gain.

\section{CONCLUSION}

In this paper we have analyzed the performances of a dual-hop relay system operating in the Ricean fading environment. The general case of non-identical fading parameters on SR and RD links, having unbalanced average SNRs per hop, is assumed. An AF relaying system with VG amplification of the received signal at $R$ is considered. A novel analytical model for performance evaluation of such a system is presented. Two essential statistical functions: PDF and MGF, describing the system end-to-end SNR, are derived in semi-closed forms. Furthermore, expressions for outage probability and bit error rate are also given. Although the obtained results are presented as an infinite sum, it is shown that it converges fast. The exact match between the analytical and simulation results is obtained using only 20 summation terms. It is noted that in the case of a higher $K$ more summands are needed.

The obtained results show that the existence of LoS between $\mathrm{S}, \mathrm{R}$ and $\mathrm{D}$ can significantly improve system performances and increase the reliability of signal transmission. Further on, this implies a special attention when the position of a relay node is planned.

The results presented in this paper prove that the performances of a dual-hop relay system are limited with the performances of a worse channel. Therefore, our future research will be focused on additional techniques, such as multi antenna nodes, that can be considered as a solution to further improve the overall system performances.

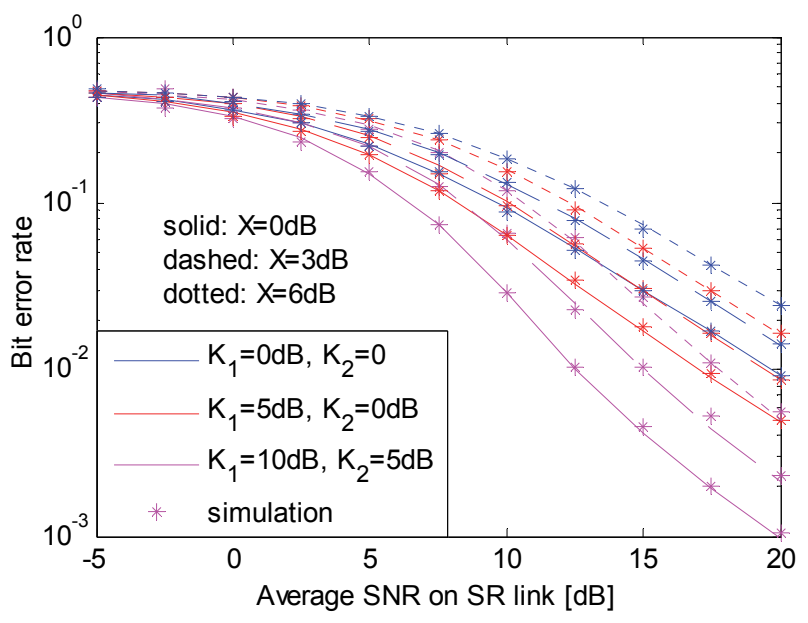

Fig. 7. BER of relay system, non-identical channels.

\section{REFERENCES}

[1] J. N. Laneman, D. N.C. Tse, G. W. Wornell, "Cooperative diversity in wireless networks: Efficient protocols and outage behavior," IEEE Trans. Inform. Theory, vol. 50, pp. 3062.3080, Dec. 2004.

[2] M. O. Hasna, M. S. Alouini, "A performance study of dual-hop transmissions with fixed gain relays," IEEE Trans. Wireless Commun., vol. 3, pp. 1963.1968, Nov. 2004.

[3] G. K. Karagiannidis, "Performance bounds of multihop wireless communications with blind relays over generalized fading channels," IEEE Trans. Wireless Commun., vol. 5, pp. 498.503, Mar. 2006.

[4] H. Mheidat, M. Uysal, "Impact of receive diversity on the performance of amplify-and-forward relaying under APS and IPS power constraints," IEEE Commun. Lett. vol. 10, pp. 468-470, June 2006.

[5] M. O. Hasna, M. S. Alouini, "End-to-end performance of transmission systems with relays over Rayleigh-fading channels," IEEE Trans. Wirel. Commun., vol. 2, pp. 1126-1131, Nov. 2003.

[6] H. A. Suraweera, G. K. Karagiannidis, "Closed-form error analysis of the non-identical Nakagami-m relay fading channel," IEEE Comm. Lett., vol. 12, No. 4, April 2008.

[7] H. A. Suraweera, R. H.Y. Louie, Y. Li, G. K. Karagiannidis, B. Vucetic, "Two hop amplify-and-forward transmission in mixed Rayleigh and Rician fading channels," IEEE Commun. Lett., vol. 13, No. 4, pp. 227-229, April 2009.

[8] J. Adeane, M. R. D. Rodrigues, I. J. Wassell, "Characterization of the performance of cooperative networks in Rician fading channels," in Proc. 12th Int. Conf. Telecomm. , Cape Town, South Africa, May 2005.

[9] T. Q. Doung, H. Shin, E. K. Hong, "Effect of line-of-sight on dualhop non regenerative relay wireless communications," in Proc. IEEE Veh. Tech. Conf. (VTC2007 Fall), Baltimore, MD, Sept/Oct. 2007, pp. 571-575

[10] M. K. Simon and M. Slim Alouini, Digital Communication over Fading Channels, Wiley-Interscience, 2005

[11] I. S. Gradshteyn and I. M. Ryzhik, Table of Integrals, Series and Products, San Diego, CA: Academic Press, 2007

[12] M. Ilic-Delibasic, M. Pejanovic-Djurisic, "Complete Analytical Model of Dual-Hop Relay System over Ricean Fading Channels," in Proc. WPMC 2013, Atlantic City, New Jersey, USA, June 2014

[13] G. Farhadi, N. C. Beaulieu, "On the ergodic capacity of wireless relaying systems over Rayleigh fading channels", IEEE Trans. Wireless Comm., vol. 7, no.11., pp. 4462-4467, Nov. 2008. 